\title{
Herramientas Web 2.0 para el fortalecimiento de resolución de problemas aritméticos con enunciado verbal
}

\author{
Web 2.0 tools for strengthening the resolution of arithmetic \\ problems with a verbal statement
}

\author{
Henser-De-Jesús Polo-Pacheco \\ Institución Educativa Distrital La Milagrosa, Fe y Alegría. \\ Planta Docente. Barranquilla, Colombia. \\ $\triangle$ hansydjesus@gmail.com \\ https://orcid.org/0000-0002-7162-8714 \\ Zulma Ortiz-Záccaro \\ Universidad de la Costa CUC. Facultad de Ciencias Humanas y Sociales. \\ Departamento de Humanidades. Barranquilla, Colombia. \\ $\triangle$ zortiz1@cuc.edu.co \\ (iD) https://orcid.org/0000-0003-4973-688X
}

\section{RESUMEN}

El trabajo de investigación tuvo como objetivo general determinar la incidencia que tiene el uso de la herramienta Web 2.0, Mangus Classroom, para el fortalecimiento de la competencia Resolución de Problemas Tipo PAEV (Problemas Aritméticos con Enunciado Verbal) en Estudiantes de Básica Primaria de Barranquilla. Su orientación metodológica fue de tipo longitudinal, cuasi-experimental, explicativo y con una modalidad de campo. La muestra estuvo representada por Estudiantes de dos (2) cursos de Cuarto Grado de la Institución Educativa Distrital La Milagrosa, Fe y Alegría, seleccionados a través de un muestreo por conveniencia. Se empleó para la recolección de datos una Prueba Tipo PreTest / Post-Test. Se obtuvo, como resultado, que el Grupo Experimental A (4001), en los problemas de tipo PAEV, mostró competencias satisfactorias, mientras el Grupo Control mantuvo sus niveles con relación al Pretest, por lo que el uso de la herramienta Web 2.0 Mangus Classroom es considerada, significativamente, como una estrategia didáctica.

Palabras clave: Herramienta Web 2.0; problemas tipo PAEV; estrategias didácticas.

\section{ABSTRACT}

The general objective of the research work was to determine the incidence of the use of the Web 2.0 tool, Mangus Classroom, for the strengthening of the competence Resolution of PAEV Problems (Arithmetic Problems with Verbal Statement) in Elementary Students of Barranquilla. Its methodological orientation was longitudinal, quasi-experimental, explanatory and with a field modality. The sample was represented by Students of two (2) Fourth Grade courses of the La Milagrosa, Fe y Alegría District Educational 
Institution, selected through convenience sampling. A Test Type Pre-Test / Post-Test Test was used for data collection. It was obtained, as a result, that Experimental Group $A$ $\left(4^{\circ} 01\right)$, in the PAEV type problems, showed satisfactory competencies, while the Control Group maintained its levels in relation to the Pretest, so the use of the Web 2.0 Mangus Classroom tool is considered, significantly, as a didactic strategy.

Keywords: Web 2.0 tool; PAEV problems; teaching strategy

\section{INTRODUCCIÓN}

En la actualidad, resulta inconcebible el no tomar en cuenta la formación matemática dentro de las principales competencias básicas que todos los individuos necesitan para desenvolverse satisfactoriamente dentro de la sociedad (Ospitaletche y Martínez, 2012). Esto cobra más sentido cuando reflexionamos que, hoy en día, se vive una cotidianidad cada vez más complicada en función de la dinámica que se vive, con mayores volúmenes de información, disponibles para una creciente cantidad de personas y con más interconexiones entre los distintos espacios de la actividad y el conocimiento humano (Prado et al, 2017); pone exigencias, también, cada vez mayores, sobre la enseñanza de la matemática (Godino et al., 2017).

En este orden de ideas, se establece que, en el ámbito colombiano, el análisis curricular realizado para fundamentar los dominios evaluados en la prueba TERCE, que se aplica a los Grados $4^{\circ}$ y $6^{\circ}$ de los países Latinoamericanos y del Caribe, denominado "Aportes para la Enseñanza de la Matemática", elaborado por la UNESCO en el año 2016, permitió, conjuntamente, identificar cómo esas exigencias han permeado los documentos curriculares de la disciplina en esta región (UNESCO, 2016).

El mencionado análisis curricular, que cotejó y analizó los documentos curriculares de matemática de los países participantes en la prueba TERCE y otros materiales académicos (como textos escolares), igualmente permitió identificar como eje central de la formación en esta área de conocimiento el desarrollo de la capacidad de resolver problemas. Esta competencia supone poner en juego todas las habilidades del pensamiento de los Estudiantes y relaciona, fuertemente, el conocimiento matemático adquirido en el ámbito escolar con la vida cotidiana.

De esta manera, como aporta la UNESCO (2016), la matemática impartida en la escuela no es comprendida como un fin en sí misma, sino que se postula como un medio para lograr los fines más transversales: formar personas capaces de razonar lógicamente y de pensar críticamente, que dominan ciertos saberes o contenidos propios de esta disciplina, pero que, además, son capaces de aplicarlos en la vida cotidiana. Así, la matemática escolar se enfoca en privilegiar su aspecto formativo.

Conforme a estos planteamientos esbozados anteriormente, la preocupación nacional de la comunidad de educadores matemáticos, desde el marco de la didáctica, está centrada en el desarrollo de procesos de formación de calidad centrado en las tres prioridades planteadas por el MEN (Ministerio de Educación Nacional) en el "Foro Educativo Nacional 2014: Ciudadanos Matemáticamente Competentes", Ios cuales se enfocan en la necesidad de una educación matemática de calidad, la importancia de considerar la enseñanza de la matemática como un aporte y el rol que toman los conocimientos en matemática en una Colombia más democrática (MEN, 2014). 
De esta manera, al revisar los resultados de las pruebas PISA (Programme for International Student Assessment), el menor desempeño se registró en matemáticas, donde menos de la quinta parte $(18 \%)$ de los evaluados alcanzó el nivel Mínimo. Según sus análisis específicos, la mayoría de los Estudiantes colombianos simplemente demostraron capacidad para identificar información y llevar a cabo procedimientos matemáticos rutinarios, siguiendo instrucciones directas en situaciones explícitas, y responder a preguntas relacionadas con contextos conocidos (OCDE, 2015).

En este sentido, aporta la OCDE (2015) que dos de los principales elementos que inciden en los bajos niveles de rendimiento en las pruebas son las actitudes menos positivas hacia la escuela y el aprendizaje, donde los alumnos con un bajo rendimiento en esta área del saber suelen mostrar menos perseverancia, motivación y confianza en sí mismos en matemáticas, que los alumnos con mejor rendimiento, debido a su poca constancia e interés.

Acorde con lo anterior, la motivación es un componente de vital importancia cuando se proyecta que el rendimiento académico de un educando sea excelente, ya que un estudiante motivado aprenderá de manera más fácil y tendrá un nivel académico proporcional a ello (Correa, Muñoz, Calderón y Pahuena, 2017). Por ende, se debe inquirir acerca de los gustos y las cosas que le son atractivas, pues, de esta forma, se obtendrá un aprendizaje significativo teniendo en cuenta el carácter multifuncional de la motivación, debido a que está marcada por lo personal y el contexto.

Tomando esto en consideración, se aporta que, en la enseñanza de las matemáticas, los Docentes deben propiciar estrategias innovadoras, que estimulen la iniciativa, creatividad e interés del estudiante, que le permita integrar la matemática con la realidad y con otras áreas del saber, por lo que el uso de materiales atractivos y estimulantes para el niño debe ser condición necesaria para apoyar este proceso (Pichardo y Puente, 2012).

Uno de los mayores retos para la formación del conocimiento en matemáticas es la Resolución de Problemas Tipo PAEV, sobre los cuales Puig y Cerdán (1988) y Guzmán (2018) consideran que un problema aritmético verbal, en adelante PAEV, es un problema de contenido aritmético que se expresa o enuncia en un contexto de información verbal.

De la misma manera, Martínez (1995) aporta que un PAEV es un problema de contenido aritmético que se expresa o enuncia en un contexto de información verbal o gráfica, atendiendo al número de datos que aparecen, explícita o implícitamente, en la información se puede hablar de PAEV simples (de una etapa) y compuestos (de varias etapas). De este modo se destaca que este tipo de problemas han sido clasificadas por diferentes autores, como lo son Castro (1994) y Pozo et al. (1994), en dos grandes categorías:

- Dificultades Sintácticas: Las dificultades sintácticas tienen que ver, en general, con la forma en la que viene enunciado el problema. Por ejemplo, se pueden nombrar las expresiones simbólicas o longitud del enunciado.

- Dificultades Semánticas: Son los significados de las distintas expresiones en el enunciado y su relación con los conceptos y procedimientos propios de la matemática.

Del mismo modo, en los últimos años las Tecnologías de la Información y la Comunicación (TIC) han tomado un gran auge en el ámbito educativo, siendo una buena opción para la actualización académica, que, además, se encuentra al alcance de todos, ganando terreno en el desarrollo de aprendizajes, debido a su carácter lúdico, que facilita la interiorización de conocimientos de una forma más divertida, generando una experiencia positiva en el alumno (Graells, 2013; Caro, 2010). 
Estas tienen una gran influencia sobre las dinámicas de la clase y el proceso de aprendizaje de los Estudiantes (Bustos y Coll, 2010), así como también permiten promover la expresión, tanto de Estudiantes como de los Docentes, para aprovecharlas como instrumento didáctico (Marqués, 2012).

El uso de las TIC se adapta especialmente bien a esta materia: la utilización de imágenes, gráficas, hojas de cálculo, etc., en calculadoras y ordenadores, permite avanzar con suma rapidez y, lo más importante, comprender y retener la información necesaria. Asimismo, las TIC abren la posibilidad de crear nuevos ambientes de aprendizaje $y$, por tanto, de desarrollar nuevas metodologías que permitan aprovechar al máximo los recursos de los que disponemos.

Según Arrieta (2013), las TIC permiten al alumnado interactuar con las matemáticas, a través de su comprensión y aprendizaje. Las metodologías asociadas al uso de TIC en el aula de matemáticas, comparten entre sí el hecho de fomentar que los Estudiantes experimenten, manipulen, corrijan, conjeturen, etc. Las TIC ponen a disposición de los Estudiantes verdaderos 'laboratorios de matemáticas' en los que conceptos matemáticos muy abstractos se materializan y el estudiante experimenta con ellos. Con lo dicho hasta ahora, parece que es imparable la modificación paulatina en la forma de enseñar las matemáticas usando las TIC.

Siguiendo esta secuencia de planteamientos, se destaca que, en el departamento del Atlántico, más específicamente en la ciudad de Barranquilla, se han impulsado distintos espacios de capacitación y formación Docente, para dar uso de las TIC como medio de apoyo en la labor Docente y convivencias.

Sobre este contexto local, se aporta que en la IED La Milagrosa, Fe y Alegría se han desarrollado propuestas interdisciplinarias que atendieron, en su momento, a distintas necesidades educativas del alumnado, pero sin tomar en cuenta el área de las matemáticas. Por lo anterior mencionado, se identifica la necesidad de implementar herramientas de las TIC para la promoción de estas en la institución.

En este sentido, el presente artículo de investigación se propone determinar la incidencia que tiene el uso de la herramienta Web 2.0 Mangus Classroom en el fortalecimiento de la competencia Resolución de Problemas Tipo PAEV (Problemas Aritméticos con Enunciado Verbal) en los Estudiantes de Grado $4^{\circ}$ de la IED La Milagrosa, Fe y Alegría. Se aporta que la herramienta Web 2.0 Mangus Classroom fue seleccionada para este estudio, ya que esta se basa en el principio de la gamificacion para generar una motivación en los Estudiantes, en el proceso de adquisición de los conocimientos (Mangus, 2018).

De la misma manera, a partir de la teoría, se presenta la hipótesis de que, a partir del proceso de intervención en la población objeto de estudio, se hallarán mayores niveles de desempeño en la Resolución de Problemas Tipo PAEV.

\section{MÉTODO}

Diseño. Desde la visión metodológica, el presente estudio se construyó desde el enfoque cuantitativo, con un diseño longitudinal, pues se llevaron a cabo las mediciones de la investigación en dos momentos: previo a la a intervención y posterior a esta.

De la misma manera, es cuasi-experimental, ya que se llevó a cabo un proceso de intervención en la población objeto de estudio, es decir, que se llevó a cabo un proceso de manipulación de las variables, sin un proceso de aleatorización de los grupos a 
intervenir. Siendo este también un estudio explicativo con una modalidad de campo, pues todo el proceso fue llevado a cabo en el lugar natural del fenómeno objeto de estudio.

Participantes. La población de estudio de esta investigación está conformada por 157 Estudiantes de Grado $4^{\circ}$ de Educación Básica Primaria de la Institución Educativa Distrital La Milagrosa, Fe y Alegría, distribuidos en 4 grupos. Los grupos de 4001 y 02 , cuentan con una cantidad de sujetos representada por 40 alumnos. Se destaca que la institución fue seleccionada por la ausencia de herramientas TIC aplicadas en el desarrollo del aprendizaje de las matemáticas.

Sobre esto, se destaca que los grupos fueron divididos por las secciones ya establecidas por la institución para facilitar el proceso de intervención dentro de la institución. De esta manera se estableció que la sección $4^{\circ} 01$ fue seleccionada como el Grupo Experimental (Grupo A) y la sección 4002 fue determinada como el Grupo Control (Grupo B), es decir, que este será el punto de comparación con el Grupo Experimental, pues a este no se le aplicará la intervención con el uso de la herramienta Mangus Classroom.

En este sentido, la presente investigación se trabajará con una muestra conformada con grupos intactos, constituidos previamente, tomando como sujetos de la investigación los 80 alumnos de los Grados 4001 y 02 que se encuentran distribuidos en igualdad numérica entre ellos. Por otra parte, con respecto a la población docente de $4^{\circ}$ de dicha institución, se determinó que la muestra estará representada por el Docente del área de matemáticas, que imparte su labor pedagógica en los cuatro grupos.

Instrumentos para la Recolección de la Información. Para generar los resultados de este estudio, se dio uso de un cuestionario Pre-Test y Post-Test aplicado a los grupos A y B. Esta prueba fue constituida por parte del Investigador, siguiendo la modalidad de cuestionario, debido a que su estructura permitió el planteamiento de 15 preguntas con múltiples opciones de respuesta, las cuales aludían a la variable a medir, Resolución de Problemas Tipo PAEV, para lo cual se plantearon distintos enunciados relacionados cada uno de ellos con una tipología de estos problemas.

Del mismo modo, se aplicó una entrevista semiestructurada, la cual contó con 15 preguntas, las cuales fueron realizadas al Docente de la institución. Otro instrumento del cual se apoyó este estudio fue la guía de observación, la cual permitió contrastar con los resultados de la entrevista formulada. Estos dos instrumentos permitieron la identificación de los procedimientos empleados por el Docente, para poner en marcha en plan de intervención.

A continuación, se muestra el baremo conforme a las categorías que se tuvieron en cuenta, de acuerdo con los resultados obtenidos en el Pre-Test y Post-Test, donde cada una de las cinco secciones tenía una ponderación que se sumaba para llegar al total:

Tabla 1. Baremo de Interpretación de Resultados.

\begin{tabular}{cc}
\hline Porcentaje & Categoría \\
\hline $78-100$ & Avanzado \\
$52-77$ & Satisfactorio \\
$26-51$ & Mínimo \\
$0-25$ & Insuficiente \\
\hline
\end{tabular}

Fuente: Elaboración propia (2019) 
Validez y Confiabilidad del Instrumento de la Investigación. En el cuestionario tomado para efectuar la prueba estandarizada para el Pre-Test y Post-Test no fue necesario realizar una validación de contenido, debido a que los insumos o ítems que la estructuran fueron extraídos de instrumentos liberados por el Ministerio de Educación Nacional, relacionados con pruebas aplicadas a Estudiantes de Grado $3^{\circ}$, a nivel nacional, denominadas Pruebas SABER $3^{\circ}$ 2014-2017, seleccionando de estas aquellos reactivos relacionados con la tipología de problemas PAEV. Con relación al guion de entrevista semiestructurada y a la lista de chequeo, estos instrumentos fueron puestos a disposición de tres (3) expertos en el área de matemáticas, didáctica y nuevas tecnologías.

Para obtener la confiabilidad del instrumento, se empleó una prueba piloto, la cual consistió en la aplicación de los instrumentos a un grupo de 10 sujetos con características similares a la población de estudio, siendo el índice de confiabilidad de 0,807, que encuentra en un nivel Muy Alto de confiabilidad.

Análisis de Resultados. En este estudio, se llevó a cabo un análisis de datos basado en la estadística descriptiva, a través de un proceso analítico de las frecuencias, las frecuencias absolutas y sus porcentajes obtenidos a partir del cuestionario aplicado, de manera que se lograse determinar la incidencia que tiene el uso de la herramienta Web 2.0 Mangus Classroom en el fortalecimiento de la competencia Resolución de Problemas Tipo PAEV (Problemas Aritméticos con Enunciado Verbal) en los Estudiantes de Grado $4^{\circ}$ de la IED La Milagrosa, Fe y Alegría.

\section{RESULTADOS}

Los resultados de este estudio se dividen en tres etapas que corresponden a:
a) La aplicación del Pre-Test
b) La intervención a través del uso del Mangus Classroom
c) La aplicación del Post-Test

Análisis e Interpretación de los Resultados del Pre-Test. A continuación, se presentan los resultados obtenidos por parte del grupo control, el cual fue identificado como "B", de manera que se pudiese diferenciar con facilidad del Grupo Experimental:

Tabla 2. Pre-Test Grupo Control B.

\begin{tabular}{|c|c|c|c|c|c|}
\hline \multirow{2}{*}{ Tipo de PAEV } & \multicolumn{2}{|c|}{ Correcta } & \multicolumn{2}{|c|}{ Incorrecta } & \multirow{2}{*}{ Categorización } \\
\hline & $\mathbf{F a}$ & $\%$ fr & $\mathbf{F a}$ & $\%$ fr & \\
\hline De cambio & 55 & 45.8 & 65 & 54.2 & Mínimo \\
\hline De combinación & 98 & 40.8 & 102 & 59.2 & Mínimo \\
\hline De comparación & 20 & 50 & 20 & 50 & Mínimo \\
\hline Multiplicativos & 54 & 45 & 66 & 55 & Mínimo \\
\hline De división & 41 & 34.2 & 79 & 65.8 & Mínimo \\
\hline Desempeño & & 43.2 & & & \\
\hline
\end{tabular}

Fuente: Elaboración propia (2019) 
Al analizar, teniendo en cuenta los diferentes ítems de cuestionario, se puede observar que existen niveles muy bajos, a nivel general, obteniendo Mínimo en todos los casos. En cuanto al nivel de desempeño general en la Resolución de Problemas Tipo PAEV, se aprecia que este es Mínimo, puesto que solo se obtuvo 43,2\% de respuestas correctas en el grupo de Estudiantes de este grupo.

Así, se presentan los resultados obtenidos por el Grupo Experimental, el cual se identifica como " $A$ ". En este sentido se puede observar lo siguiente con los resultados obtenidos:

Tabla 3. Pre-Test Grupo Experimental A

\begin{tabular}{cccccc}
\hline \multirow{2}{*}{ Tipo de PAEV } & \multicolumn{2}{c}{ Correcta } & \multicolumn{2}{c}{ Incorrecta } & \multirow{2}{*}{ Categorización } \\
\cline { 2 - 5 } & Fa & \% fr & Fa & \% fr & \\
\hline De cambio & 45 & 37.5 & 75 & 62.5 & Mínimo \\
De combinación & 120 & 60 & 80 & 40 & Satisfactorio \\
De comparación & 14 & 35 & 26 & 65 & Mínimo \\
Multiplicativos & 44 & 36.7 & 76 & 63.3 & Mínimo \\
De división & 29 & 24.2 & 91 & 75.8 & Insuficiente \\
Desempeño & & 38.7 & & \multicolumn{2}{c}{ Mínimo } \\
\hline
\end{tabular}

Fuente: Elaboración propia (2019).

En el caso del Pre-Test para el Grupo Experimental A, es apreciable que en las diferentes categorías se obtuvieron puntajes muy bajos también, donde se observó la aparición del resultado "Insuficiente", tres de "Mínimo" y "Satisfactorio" una vez. De forma general, el nivel de desempeño en los tipos de PAEV es Mínimo, puesto que se aprecia un 38.7\% de respuestas correctas en el grupo de Estudiantes.

Proceso de Intervencion mediante la Utilización de Mangus Classroom. Para desarrollar el proceso de intervención al Grupo Experimental "A", se procedió a la ejecución de las estrategias. Se creó un Ambiente Virtual de Aprendizaje (AVA), utilizando la herramienta de Mangus Classroom, el cual tiene por nombre iAprendo, juego y resuelvo!, denominación que fue asignada por el Investigador, debido a la esencia de este espacio virtual gamificado, en donde los sujetos adquirieron nuevos conocimientos sobre los PAEV, ejercitando lo aprendido de forma lúdica, a través de diversas actividades que debían resolver para ganar distintos reconocimientos y beneficios.

Para ello, se dividió este ambiente o curso creado en seis sesiones, en las cuales se trataron temáticas específicas relacionadas con los Problemas Aritméticos de Enunciado Verbal (PAEV), conformadas estas a su vez por seis (6) instantes de trabajo, tres (3) lecciones (teórica, práctica y evaluativa), un espacio de discusión o foro, una encuesta y una actividad (foro o encuesta, usada al inicio de cada sesión). Cada uno de estos instantes de trabajo, de igual forma, fueron nombrados conforme a la actividad o lección que se iba a realizar y agrupados en los tres momentos de la ruta didáctica propuestos por el MEN (2016).

De esta manera, se desarrollaron 38 actividades de forma secuencial, teniendo en cuenta la temática abordada en las sesiones. Cada una de estas actividades contaba con una puntuación asignada de forma automática por la plataforma, que alcanzaban los sujetos cuando lograban superar los retos que se planteaban en ellos, específicamente los distintos ejercicios relacionados a Ios PAEV. 
Búsqueda. 6(23):e445 Julio-Diciembre, 2019. https://doi.org/10.21892/01239813.445

Tabla 4. Estructura de las Sesiones

\begin{tabular}{ccc}
\hline & Sesión & \\
\hline Momento de la secuencia didáctica & Nombre del instante de trabajo & Actividad/lección \\
\hline Momento de exploración & Antes de & Foro o encuesta \\
Momento de estructuración y práctica & iaprende y gana! & Lección teórica \\
& iAhora te toca a ti! & Lección práctica \\
Momento de transferencia y valoración & iDiscute y aprende! & Encuesta \\
& iInteractúa y resuelve! & Foro \\
\hline
\end{tabular}

Fuente: Elaboración propia

Análisis e Interpretación de los Resultados del Post-Test. En primera instancia, se muestran los resultados obtenidos por el Grupo Control B, del Post-Test aplicado sobre Ios ejercicios relacionados a los PAEV:

Tabla 5. Post-Test Grupo Control B

\begin{tabular}{cccccc}
\hline \multirow{2}{*}{ Tipo de paev } & \multicolumn{2}{c}{ Correcta } & \multicolumn{2}{c}{ Incorrecta } & \multirow{2}{*}{ Categorización } \\
\cline { 2 - 5 } & Fa & \%fr & Fa & \%fr & \\
\hline De cambio & 68 & 56.7 & 52 & 43.3 & Satisfactorio \\
De combinación & 132 & 66.0 & 68 & 34.0 & Satisfactorio \\
De comparación & 17 & 42.5 & 23 & 57.5 & Mínimo \\
Multiplicativos & 66 & 55 & 54 & 45 & Satisfactorio \\
De división & 37 & 30.8 & 83 & 69.2 & Mínimo \\
Desempeño & & 50.2 & & \multicolumn{2}{c}{ Mínimo } \\
\hline
\end{tabular}

Fuente: Elaboración propia (2019).

De manera general, el nivel de desempeño en la resolución de los tipos de PAEV en el Grupo de Control B promedió un desempeño Mínimo, puesto que se aprecia un $50.2 \%$ de respuestas correctas en el grupo de Estudiantes. Por consiguiente, estos resultados son contrarios a lo expuesto por Martínez (1995), quien plantea que es así como estos problemas se convierten en el campo de entrenamiento, en el que los Estudiantes traducen el lenguaje ordinario a la aplicación del lenguaje matemático, pero, por los resultados, es Mínimo ese nivel de traducción.

Del mismo modo, los resultados del Post-Test, obtenido por el Grupo Experimental A, fue:

Tabla 6. Post-Test Grupo Experimental A

\begin{tabular}{cccccc}
\hline \multirow{2}{*}{ Tipo de PAEV } & \multicolumn{2}{c}{ Correcta } & \multicolumn{2}{c}{ Incorrecta } & \multirow{2}{*}{ Categorización } \\
& Fa & \%Fr & Fa & \%Fr & Satisfactorio \\
De cambio & 74 & 61.6 & 46 & 38.3 & Satisfactorio \\
De combinación & 142 & 71 & 58 & 29 & Satisfactorio \\
De comparación & 24 & 60 & 16 & 40 & Avanzado \\
Multiplicativos & 94 & 78.3 & 26 & 21.7 & Mínimo \\
De división & 59 & 49.2 & 61 & 50.8 & \\
Desempeño & & 64.02 & & & Satisfactorio \\
\hline
\end{tabular}

Fuente: Elaboración propia (2019) 
En general, el nivel de desempeño evidenciado en el Grupo Experimental A, en la resolución de los tipos de PAEV, es Satisfactorio, puesto que se aprecia un $64.02 \%$ de respuestas correctas, resultado que logra ubicarlos en este nivel, según el baremo.

\section{DISCUSIÓN}

Después de aplicada la herramienta Web 2.0 Mangus Classroom, para el desarrollo de una serie de sesiones que corresponden específicamente a una tipología PAEV, y dentro de las cuales se desarrollaron distintas estrategias didácticas organizadas en una secuencia estructurada por los tres momentos que plantea en MEN (2014), ya explicados en la matriz presentada en páginas anteriores, se aplicó nuevamente la misma prueba estandarizada, realizada al inicio de la investigación, pero, en esta ocasión, con una connotación distinta, debido a que su aplicación estaba centrada en establecer la diferencia del nivel general de desempeño de los sujetos del Grupo A, con respecto a los del grupo B, después de realizadas dichas sesiones mediadas por esta TIC.

De esta forma, los resultados generados por este instrumento, permitieron establecer una mejora en el desempeño de los alumnos del grupo A quienes fueron participes de estas sesiones usando la plataforma mencionada anteriormente, a diferencia de los sujetos del grupo B, los cuales continuaron con sus clases tradicionales sin recibir este tratamiento, comprobando de esta manera la hipótesis de investigación y rechazar la hipótesis nula.

En los resultados que se reflejan en la Tabla 5, en relación a Ios PAEV de Cambio, más precisamente los porcentajes de respuestas correctas, el $61.6 \%$ (Grupo Experimental A), se percibe notoriamente una mejoría en el desempeño de los sujetos mencionadas anteriormente, en razón de que lograron asociar la cantidad inicial y la disminución que esta sufre en sí para determinar la cantidad final.

Este resultado plantea una reflexión sobre lo expuesto por Bermejo (1990), al exponer que los problemas de cambio se caracterizan por la presencia de una acción explícita o implícita, que modifica una cantidad inicial, dando como resultado el incremento o decremento de esa cantidad.

De la misma forma, en los ítems relacionados con los PAEV de Combinación, se aprecia en dicha Tabla un gran porcentaje de opciones correctas seleccionadas por parte de los sujetos, más precisamente el 71\% (Grupo Experimental A), evidenciándose el proceso de diferenciación entre la cantidad que representa una de las partes y la cantidad total, para así determinar la cantidad que se refiere a la parte faltante.

En el resultado hay una fortaleza de acuerdo con Bermejo, (1990) puesto que estos problemas implican relaciones estáticas, en las que se proponen dos cantidades disjuntas, que pueden considerarse aisladamente o como parte de un todo, sin que haya ningún tipo de acción, logrando diferenciar la cantidad que representa una de las partes y la cantidad total, para así determinar la cantidad que se refiere a la parte faltante.

Haciendo referencia a lo que se observa en esta Tabla, con respecto a los PAEV de Comparación, más específicamente el 60\% (Grupo A) de aciertos en estas situaciones, nuevamente se aprecian fortalezas de los sujetos al momento de afrontar estos enunciados, conforme a lo mencionado por Bermejo (1990), en razón de que logran asociar la comparación de más que se establecía en la cantidad comparada con la cantidad referente, lo que los indujo a realizar una sustracción en vez de una adición para averiguar la cantidad referente. 
Con referencia a los ítems relacionados a PAEV de Estructura Multiplicativa, el porcentaje de aciertos está representado por un 78.3\% (Grupo Experimental A), resultados que reflejan la presente agrupación a todos los tipos de problemas que establecen relaciones fijas entre cantidades, de acuerdo con Schmidt y Weiser (1995).

Por último, en cuanto a los PAEV de División, 49.2\% (Grupo A) de porcentaje de respuestas correctas, permiten observar avances Satisfactorios en la solución de estas situaciones, aunque no tan significativas como las anteriores, pero sí presentándose fortalezas en los problemas de partición, razón y producto cartesiano, al diferenciar la relación entre las cantidades y su función dentro del enunciado, y la incógnita a hallar, conforme a lo expuesto por Vergnaud (1983).

De acuerdo con lo anterior, es innegable el impacto que generó el empleo de la herramienta Web 2.0, en el fortalecimiento de la competencia Resolución de Problemas Tipo PAEV, en los sujetos del Grupo Experimental A.

Se hace innegable reconocer que la flexibilidad del espacio virtual que rodea a esta herramienta, posibilitó la estructuración de una secuencia didáctica que organizó todas las sesiones trabajadas con dichos sujetos, basándose en los planteamientos de Díaz (2013) sobre las tipologías de actividades de aprendizaje, y el MEN (2016) con respecto a los momentos de una secuencia didáctica, para, de esta manera, organizar los contenidos de una forma llamativa y dinámica, conforme al instante de la ruta planteada, determinando una serie de instantes de trabajo que posibilitaran una mayor aprehensión de los nuevos saberes, así como también la práctica y evaluación de estos.

De esta manera, lo anteriormente planteado se relaciona con lo expuesto por Brousseau (1997) en su teoría sobre la situación didáctica, donde se crea un escenario, la excusa o conjunto de actividades que, en conjunto, estimulan el desarrollo de los Estudiantes.

En determinada situación se lleva a cabo una relación interactiva entre todos los participantes, incluido el Docente, quien también, inspecciona que se alcancen los contenidos dispuestos.

Es así, que las situaciones didácticas tienen por finalidad que los Discentes aprendan a través de la relación que estos tengan con el problema que se plantee, respondiendo al mismo basándose en sus conocimientos, motivados por la situación formulada y no por satisfacer el deseo del Maestro, y sin que el Docente interceda directamente, ayudándolo a encontrar una solución (Argudín, 2015).

Por último, al haber identificado el elemento motivacional, dentro del aula de clases, como elemento diferenciador en el desarrollo de los conocimientos del alumnado, se puede aseverar, entonces, que se deben realizar futuros estudios que permitan medir el factor motivacional de la herramienta Mangus Classroom para medir sus efectos dentro de este factor.

Financiación. Este artículo es producto del proyecto desarrollado, titulado "Resolución de Problemas Aritméticos de Enunciado Verbal (PAEV), mediante el uso de Mangus Classroom en Estudiantes de Grado $4^{\circ}$ de la IED Milagrosa, Fe y Alegría de Barranquilla". 


\section{REFERENCIAS}

Arrieta, J. E. (2013). Las TIC y las matemáticas, avanzando hacia el futuro. Universidad de Cantabria. http://bit.ly/2Vtluch

Bermejo, V. (1990). El niño y la aritmética. Instrucción y construcción de las primeras nociones aritméticas. Paidos

Brousseau, G. (1997). Théorie des situation didactiques et ses applications. http://bit. Iy/2L8SJ1D

Argudin, Y. (2015). Educación basada en competencias. Magistralis, 20, 39-61.

Bustos, A. y Coll, C. (2010). Los entornos virtuales como espacios de enseñanza aprendizaje. Una perspectiva psicoeducativa para su caracterización y análisis. Revista Mexicana de Investigación Educativa, 15(44), 163-184. http://bit.ly/2WdLvKO

Castro, E. (1995). Niveles de comprensión en problemas verbales de comparación multiplicativa. COMARES.

Correa, A., Parra, M., Calderón, L., Pahuena, D. (2018). Bajo rendimiento académico en el área de matemática de Estudiantes del grado tercero del colegio integrado de Cabrera sede C Escuela Rural el Diamante (Trabajo de grado de Licenciatura). http:// bit.ly/2XVIRuK

Godino, J. D., Giacomone, B., Batanero, C., Font, V. (2017). Enfoque ontosemiótico de los conocimientos y competencias del profesor de matemáticas. Boletim de Educação Matemática, 31(57), 90-113.

Graells, P. M. (2013). Impacto de las TIC en la educación: funciones y limitaciones. 3C TIC, 2(1).

Guzmán, A. (2018). Nivel de resolución de problemas aditivos (PAEV) en Estudiantes de dos instituciones educativas de San Juan de Lurigancho-2018.

Instituto Colombiano para la Evaluación de la Educación (ICFES) (2015). Informe Ejecutivo sobre resultados de Colombia en PISA. http://bit.ly/2DTTN3v

Mangus Classroom. (2018). Gamificación en la educación. https://mangus.org/blog/94gamificacion-en-la-educacion.

Marqués, P. (2012). Modelos didácticos de aprendizaje en grupo y aprendizaje colaborativo con TIC. Comunicación y pedagogía: Nuevas tecnologías y recursos didácticos, (261), 26-29.

Martínez M. J. (1995). Importancia de los PAEV de una etapa: algunas indicaciones para su tratamiento en el aula. Revista de Ciencias de la Educación, 12, 169-184

Ministerio de Educación Nacional (MEN). (2014). Documento Orientador. Foro Educativo Nacional: Ciudadanos Matemáticamente Competentes. http://bit.ly/2WnVBca

Organización para la Cooperación y el Desarrollo Económico (OCDE) (2016). Education in Colombia. OCDE 
Organización para la Cooperación y el Desarrollo Económicos (OCDE) (2016). PISA 2015 resultados claves. http://bit.ly/2PFOatc

Ospitaletche, E., Martínez, V. (2012). La Matemática como idioma y su importancia en la enseñanza y aprendizaje del Cálculo. Números. Revista de Didáctica de las Matemáticas, 79, 7-16.

Pichardo, I. M. C., Puente, Á. P. (2012). Innovación Educativa: Uso de las TIC en la enseñanza de la Matemática Básica. EDMETIC, 1(2), 127-144.

Pozo, J. I., Pérez, M. D., Domínguez, J., Gómez, M. A., Postigo, Y. (1994). La solución de problemas. Santillana.

Prado, S., Arregui, E., Martín, A., Fraga, I., Agudo, S., Álvarez-Arregui, E., Rosal, I. (2017). Envejecer en el umbral del siglo XXI: una sociedad TIC. In Innovación Docente y uso de las TIC en educación: CD-ROM (p. 92). Universidad de Málaga (UMA).

Puig, L., Cerdán, F. (1988). Problemas aritméticos escolares. Síntesis

Schmidt, S., Weiser, W. (1995). Semantic structures of one-step word problems involving multiplication or division. Educational Studies in Mathematics, 28, 55-72. 